\title{
TARIFFS IN ENERGY: CURRENT FACTORS AND THE CONCEPT OF CHANGE
}

\section{DEMUR CHOMAKHIDZE}

Doctor of Economic Sciences, Professor

Georgian Technical University,

Academician of Academy of Economic Sciences of Georgia, Georgia

d.chomakhidze@gnerc.org

Abstract. Factors acting on tariffs are characterized including the state of utilization of existing natural energy resources, the structure of electricity generation, energy efficiency, energy balance, the role of society in energy development and globalization.

It has been concluded that in Georgia, as well as in the world, there is a tendency to increase tariffs. This is due to the need for increased and continuous investment in the extraction (production) of increasing amounts of energy resources, the tightening of environmental requirements, and so on.

According to the author, in order to improve the technical and economic indicators in the field and reduce tariffs, it is necessary to introduce scientific-technological and managerial progress at a higher rate compared to the existing challenges.

\section{KEYWORDS: GEORGIA, ENERGY, TARIFF, ELECTRICITY, NATURAL GAS.}

For citation: Chomakhidze, D., (2021). Tariffs in Energy: Current Factors and the Concept of Change. Globalization and Business, №11, 87-91. (In Georgian). https://doi.org/10.35945/gb.2021.11.011 


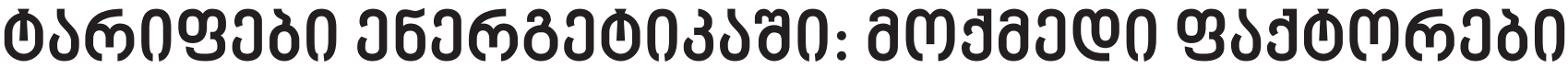

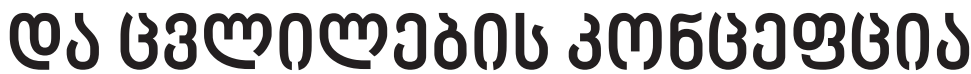

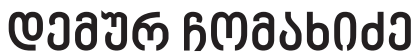

गзलбмдаз

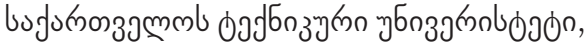

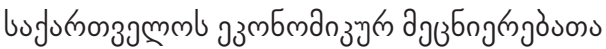

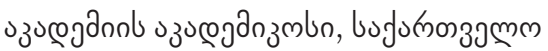

d.chomakhidze@gnerc.org

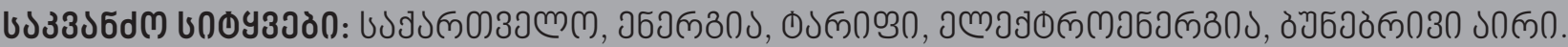

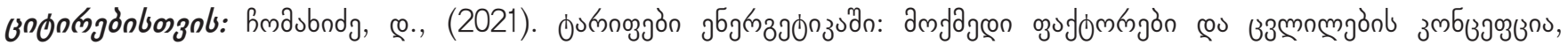

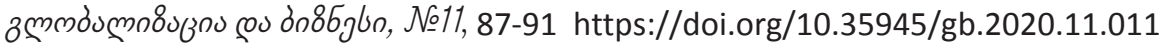

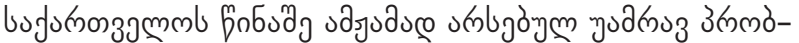

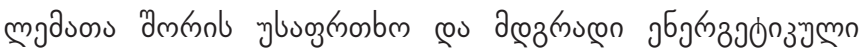

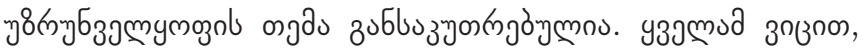

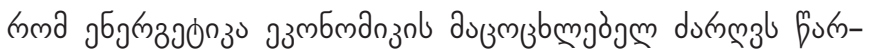

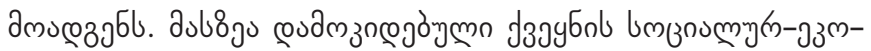

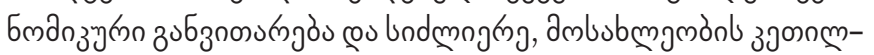

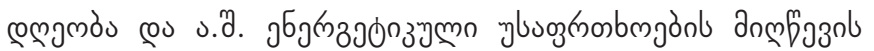
зง

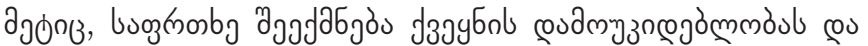

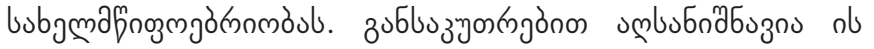

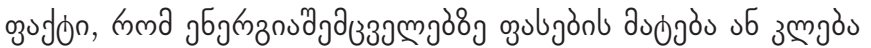

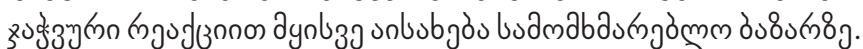

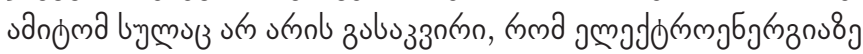

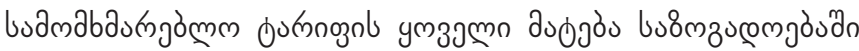

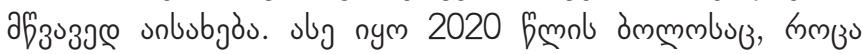

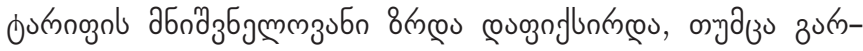

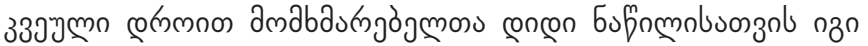

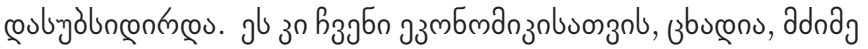
Ozonomos.

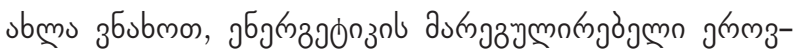

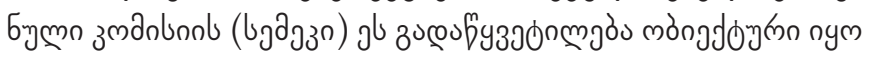
ond ono.

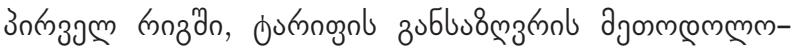

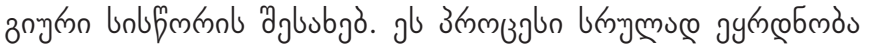

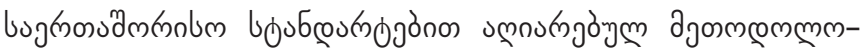

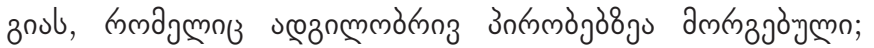

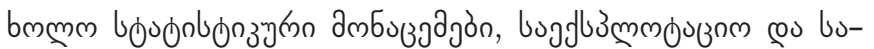
nб

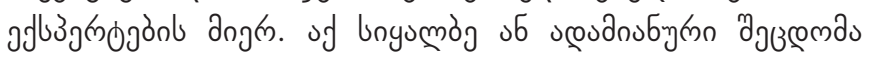

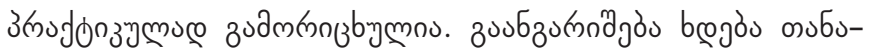

agn

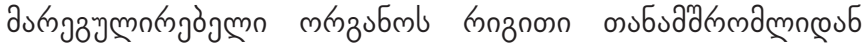

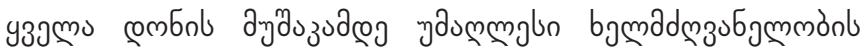

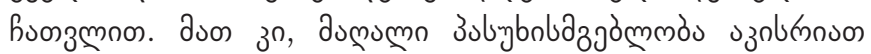

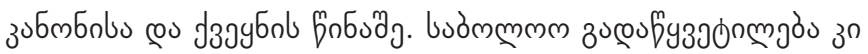

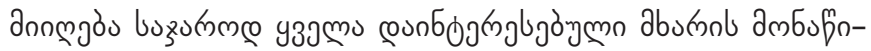

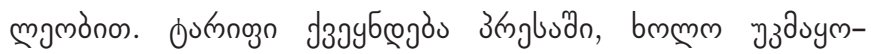

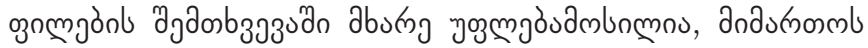

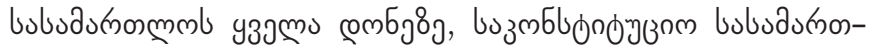

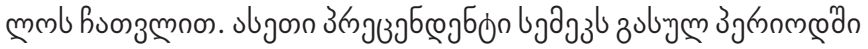

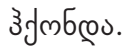

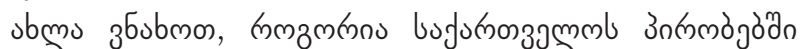

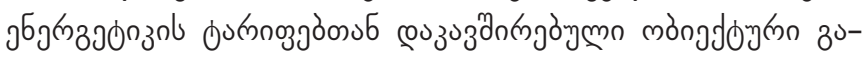

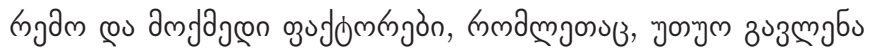
ufzon jumnognl combnl zublus mz

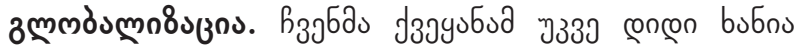

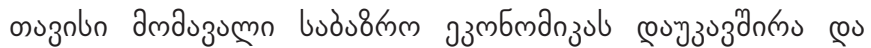

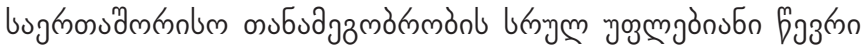

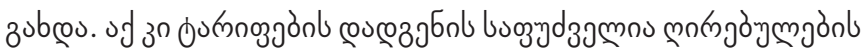

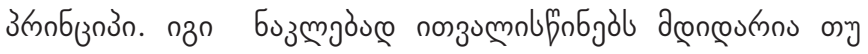

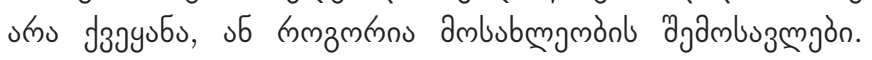

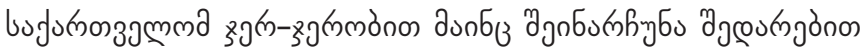

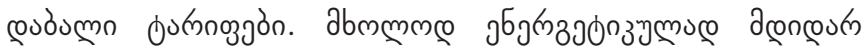

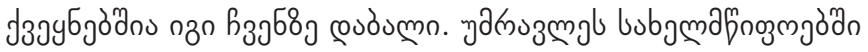

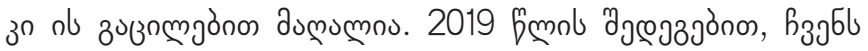

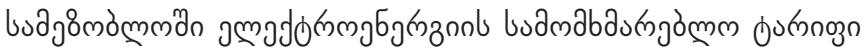
ubgon nym:

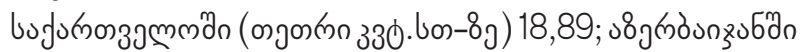

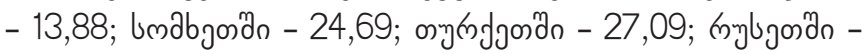
16,34 . 


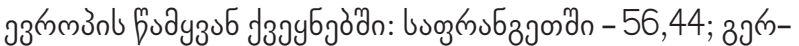

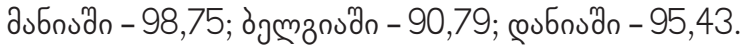

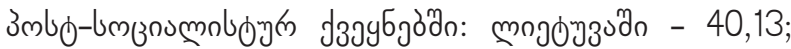

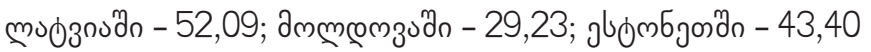
cos o. .

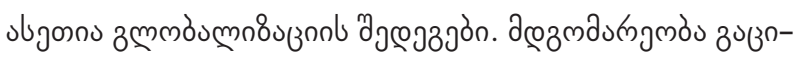

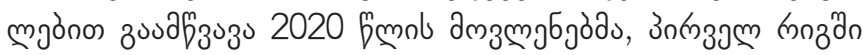

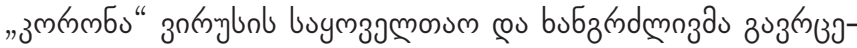

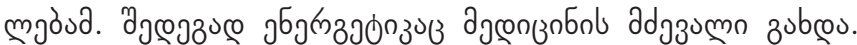

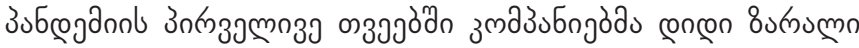

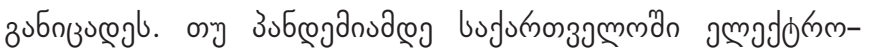

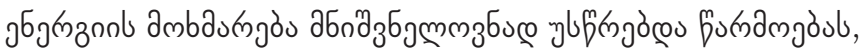

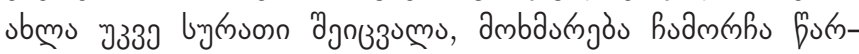

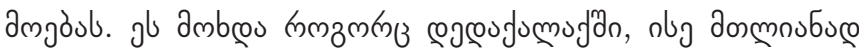
bufunonsammañ.

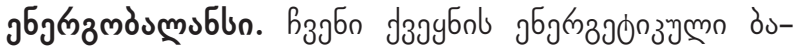

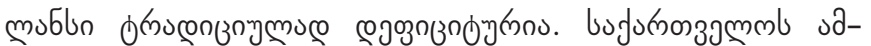

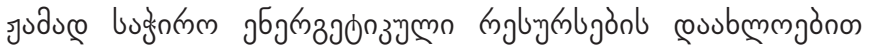

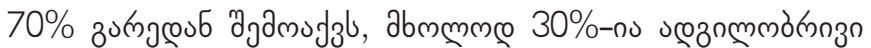
furnamgönl. jбgr

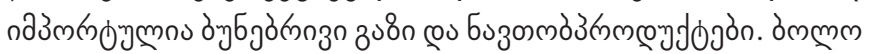

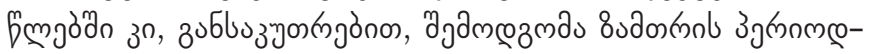

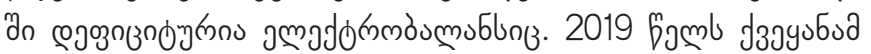

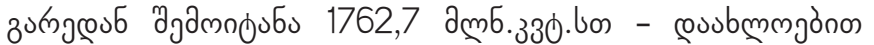

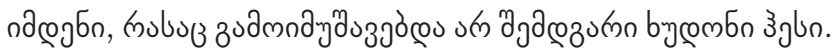

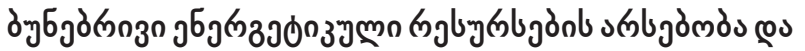

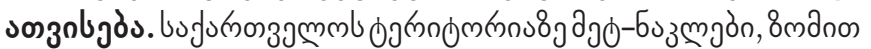

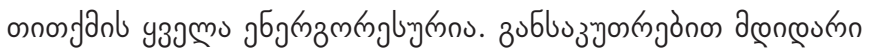

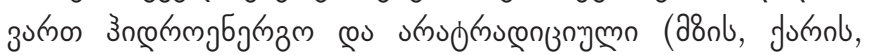

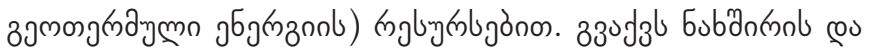

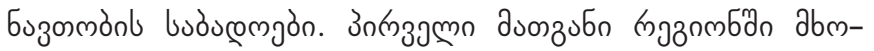

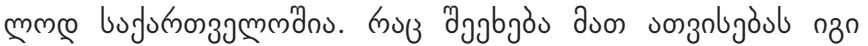

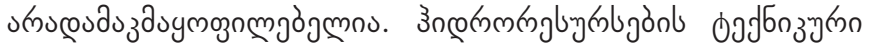

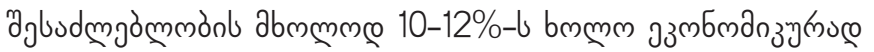

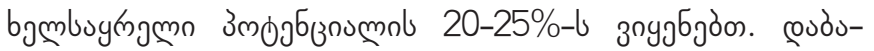

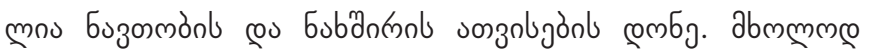

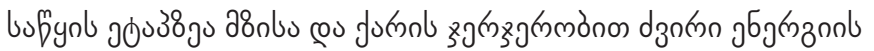
zodmgjбjò.

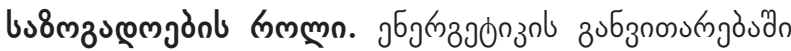

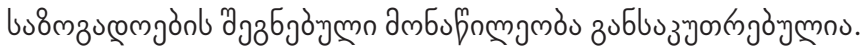

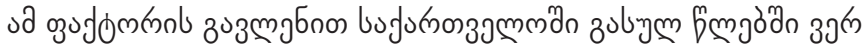

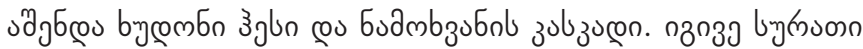

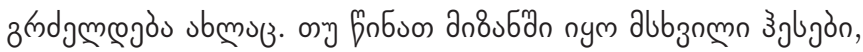

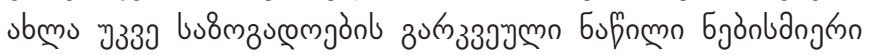

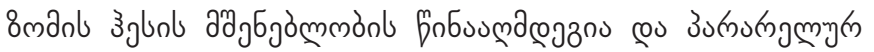

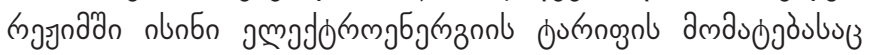

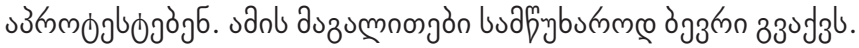

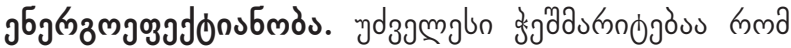

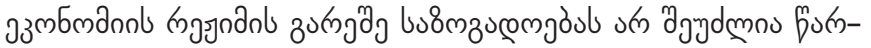

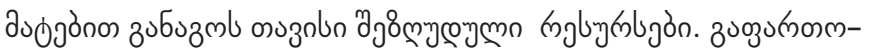

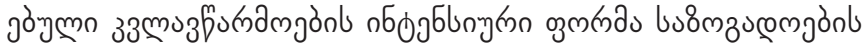

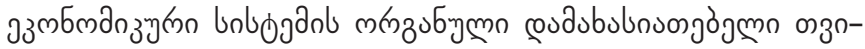

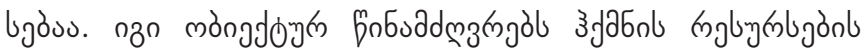

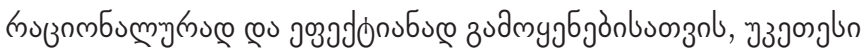

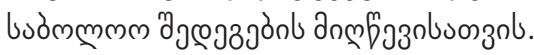

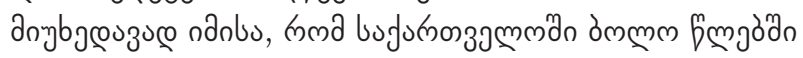

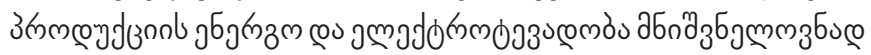

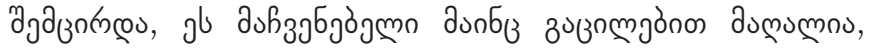

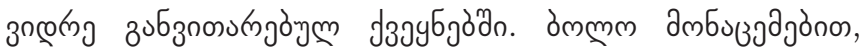

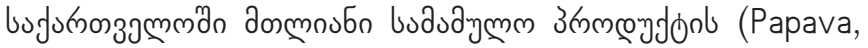

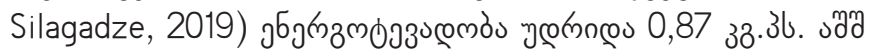

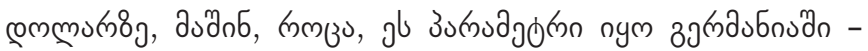

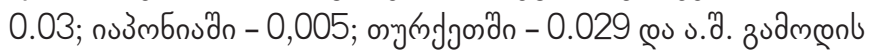

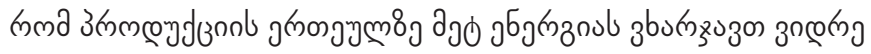
jбgr

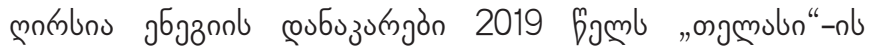

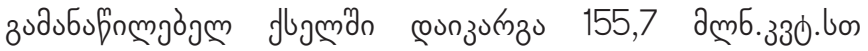

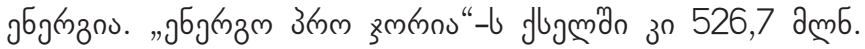

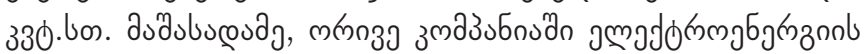

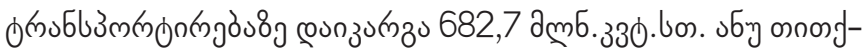

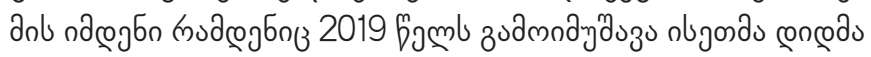

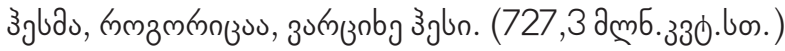

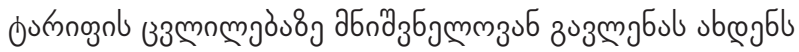

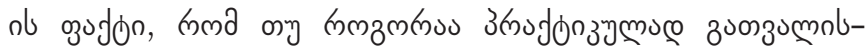

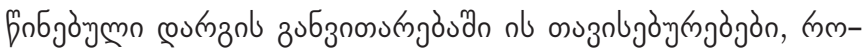

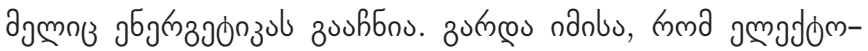

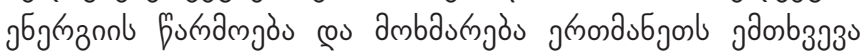

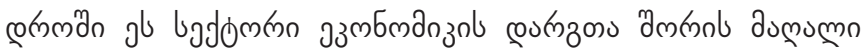

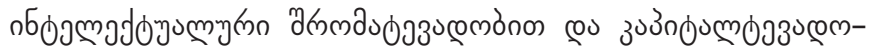

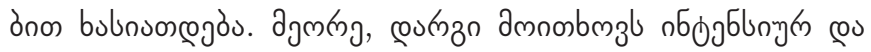

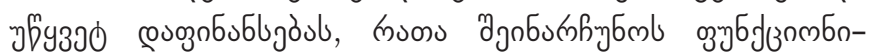

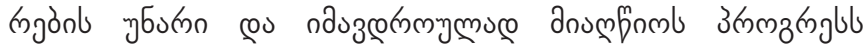

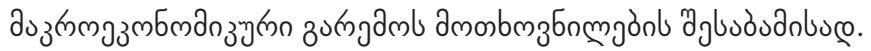

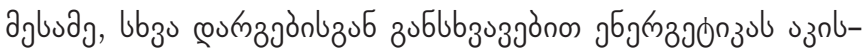

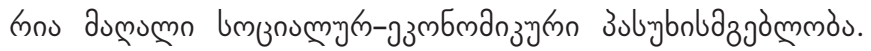

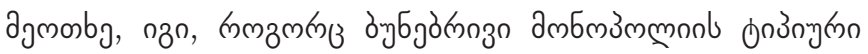

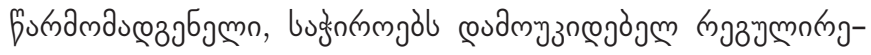

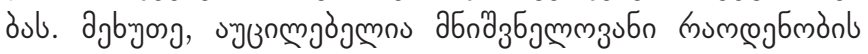

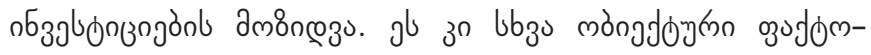

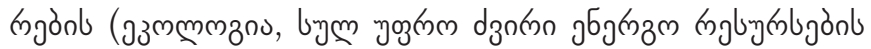

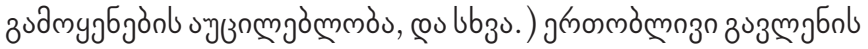

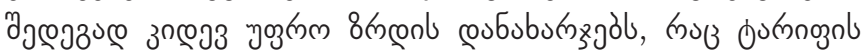

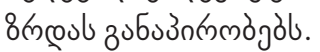

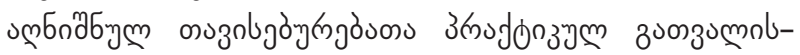

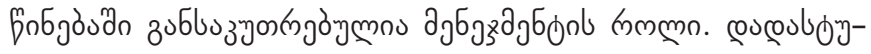

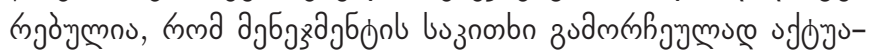

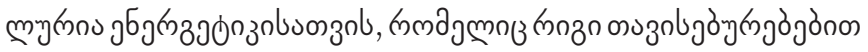

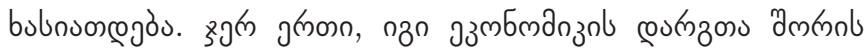

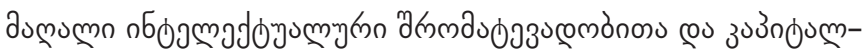

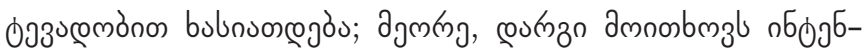




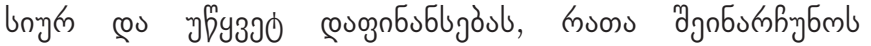

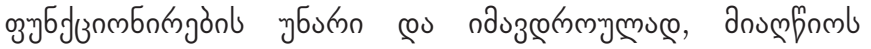

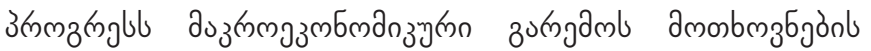

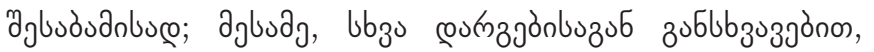

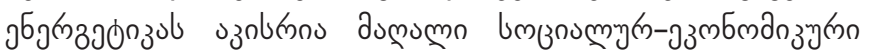

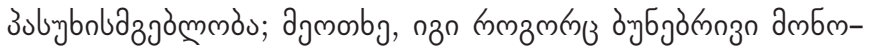

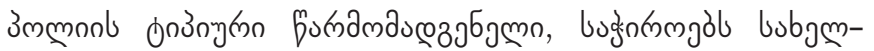

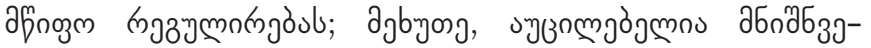

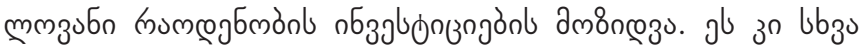

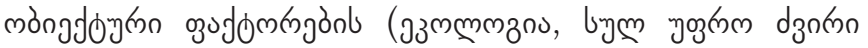

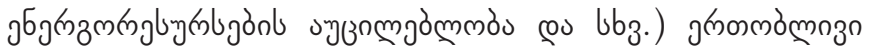

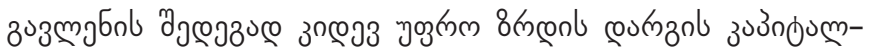

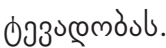

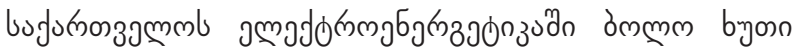

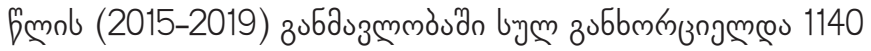

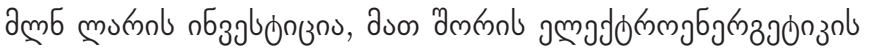

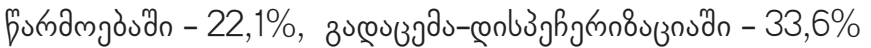

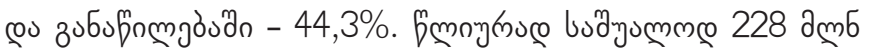
motion.

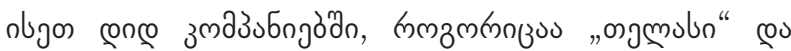

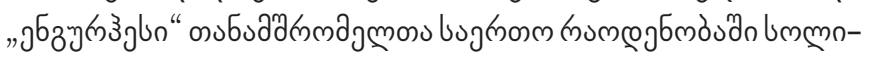

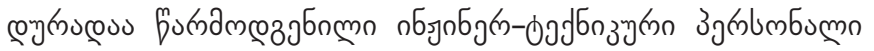

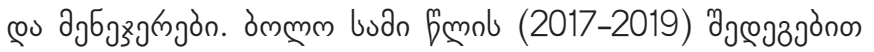

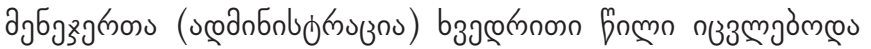

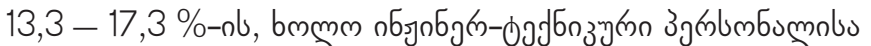

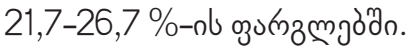

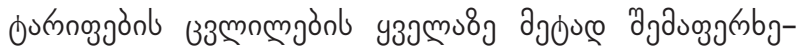

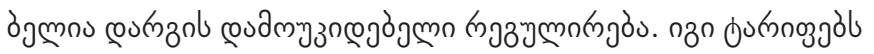

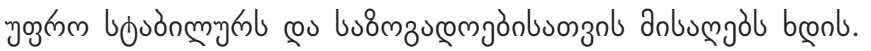

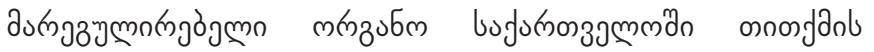

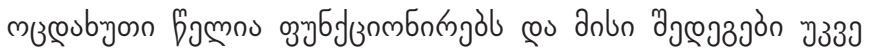

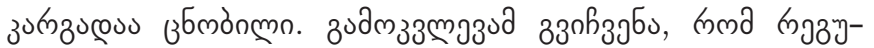

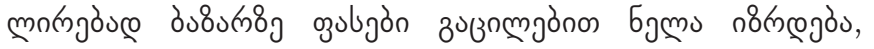

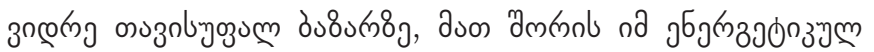

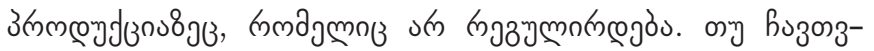

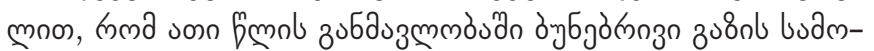

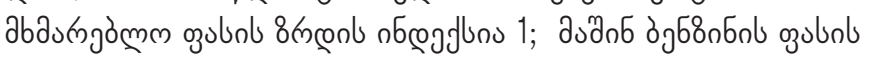

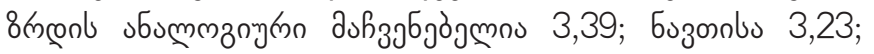

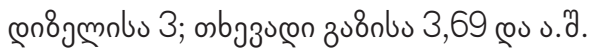

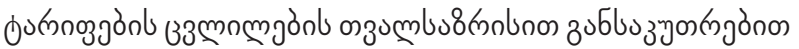

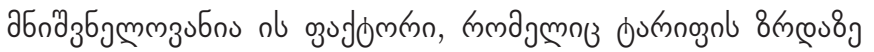

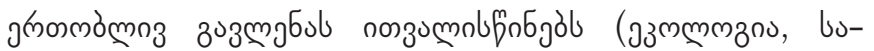

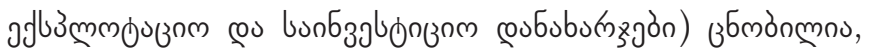

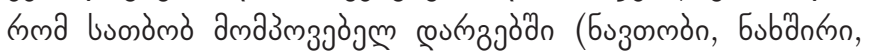

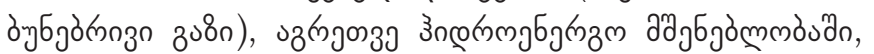

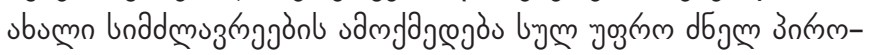

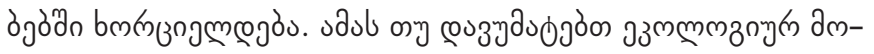

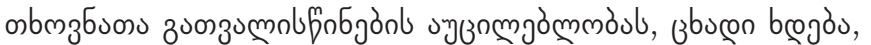

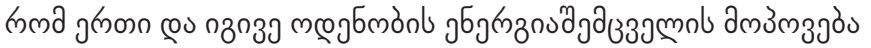

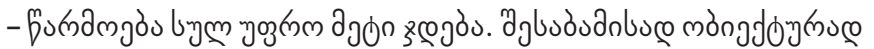

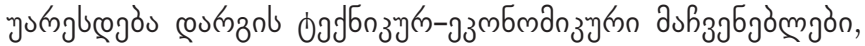

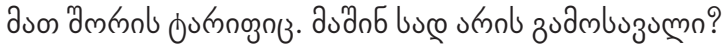

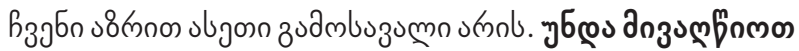

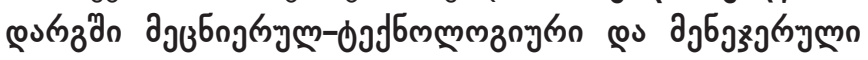

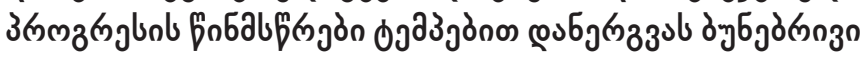

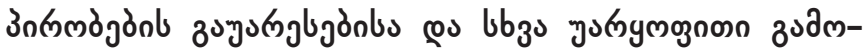

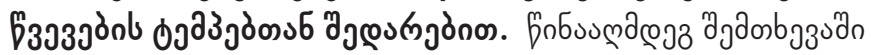

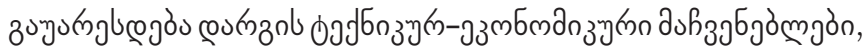

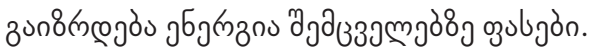

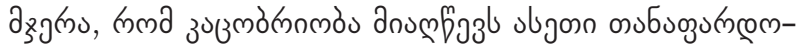

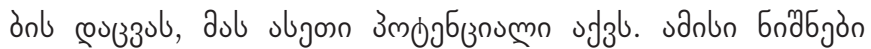

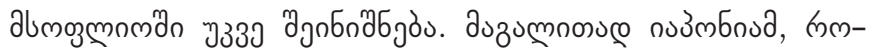

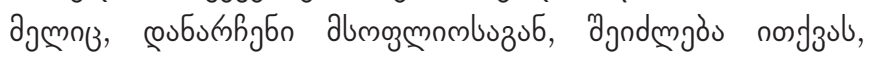

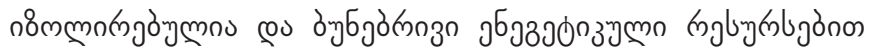

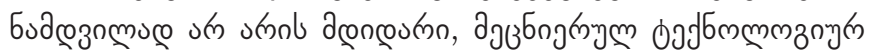

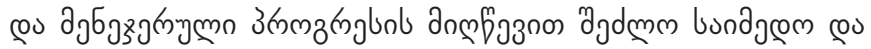

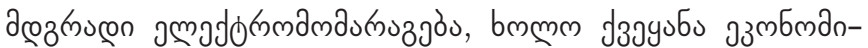

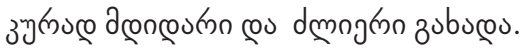

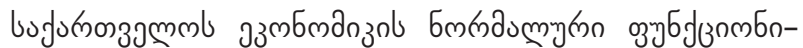

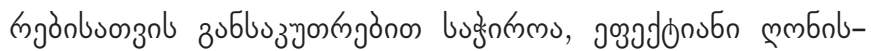

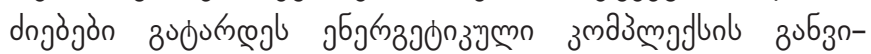

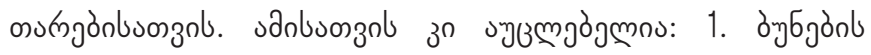

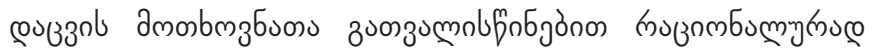

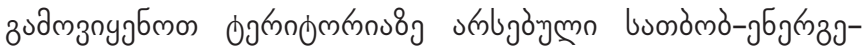

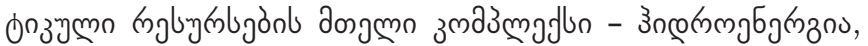

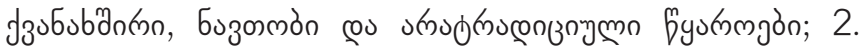

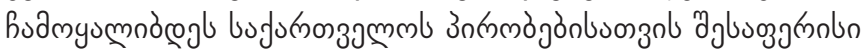
buos̀mo-gб

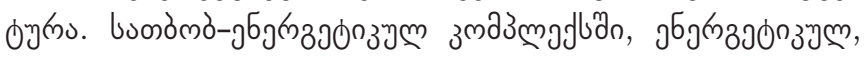

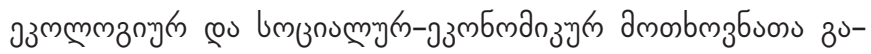

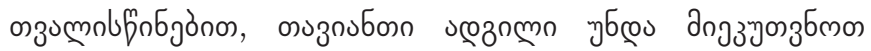

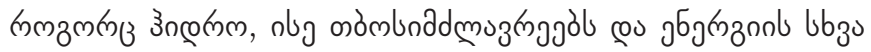

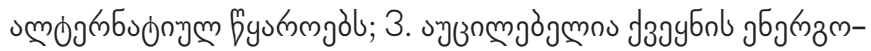

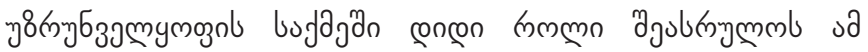

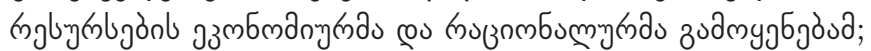

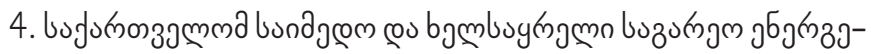

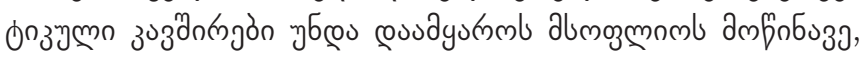

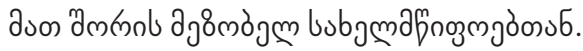

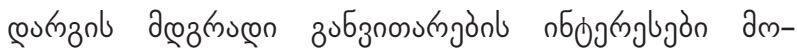

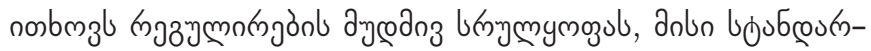

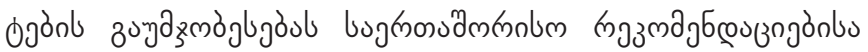

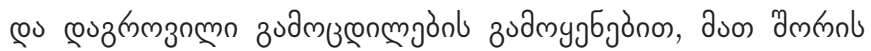

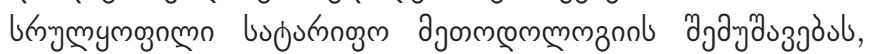

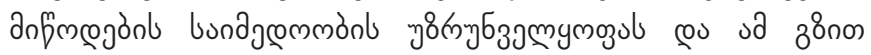

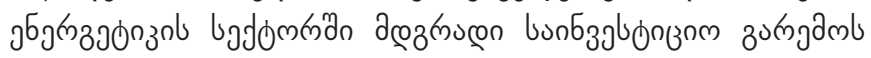

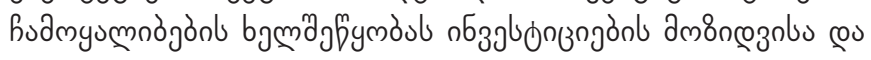

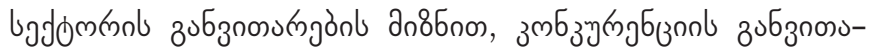

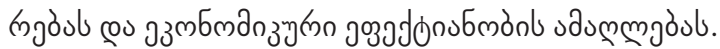

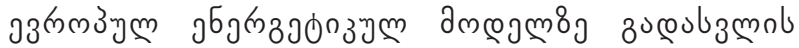

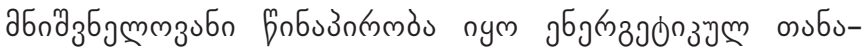




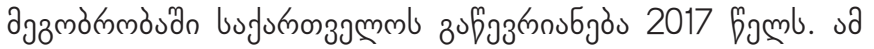

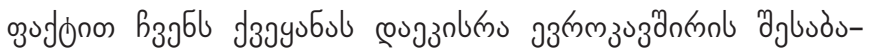

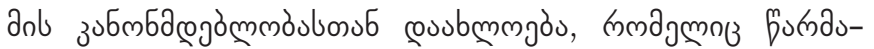

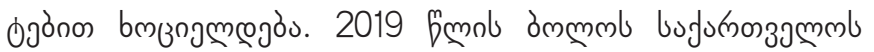

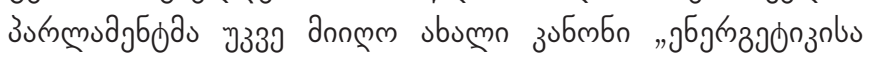

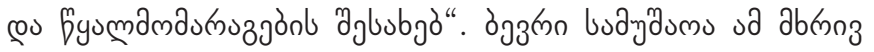

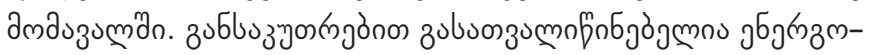

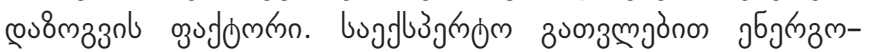

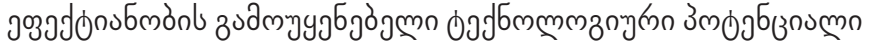

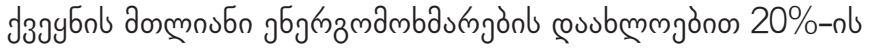
ommns. sаূ̣

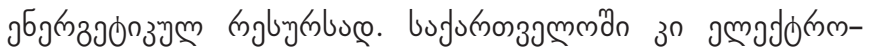

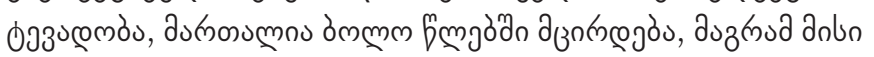

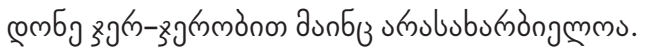

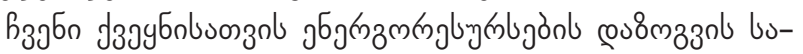

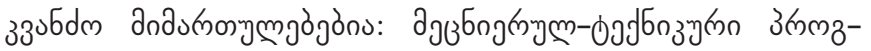

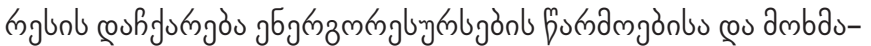

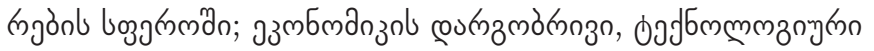

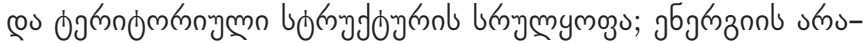

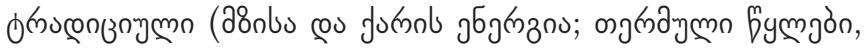

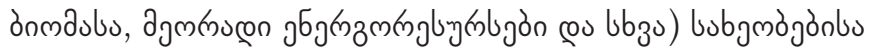

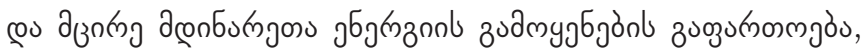

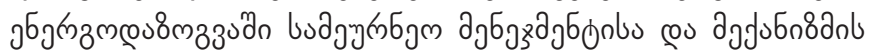
uñymymoुs.

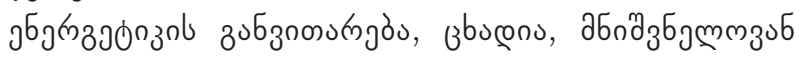

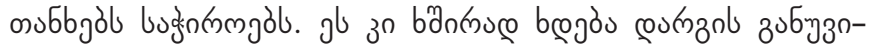

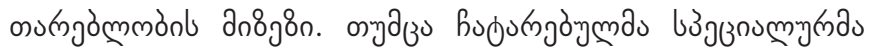

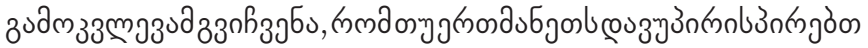

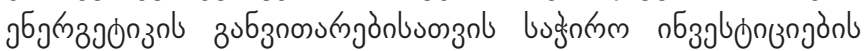

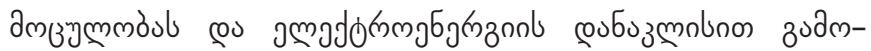

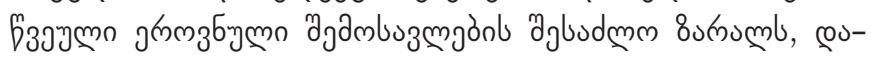

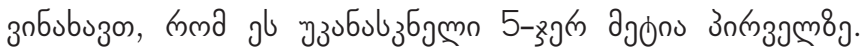

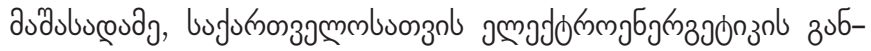

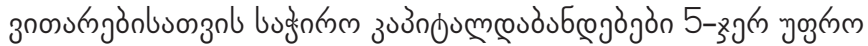

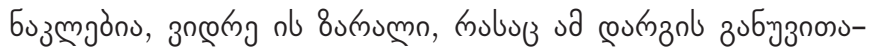

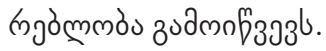

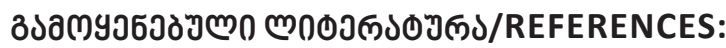

Aspects, Proceedings of the Third National Scientific National Conference.

Chomakhidze, D., (2016). energy balance of Georgia."ELSEVIER" AASCI.

Chomakhidze, D., (2016). Georgian Energy in the World Energy System. "Globalization and Business, N-2 2016.

Chomakhidze, D., Melikidze, M., (2019) - Methodological and Conceptual Basis for Energy Development in Georgia, Lambert academic publishing.

Chomakhidze, D., Kublashvili, G., Mosakhlishvili, L., (2018) Renewable Energy of Georgia: Sources and Realization. LAMBERT academic publishing.

Geostat - Energy Balance 2013-2019.

GEWRC - Annual Report 2019.

IEA - “Key World Energy Statics" 2019.

Law of Georgia on Energy and Water Supply, 2019.

Mirtskhulava, D., Chomakhidze, D. , Tsintsadze, P., Eristavi, E., Arveladze, R., and others - Georgia's energy strategy. Bakur Sulakauri Publishing House, 2004.

Narmania, D. Chomakhidze D., (2019). Georgian Energy Balance in the Years of Independence. Globalization and Business, N-9.

Narmania, D., \& Chomakhidze, D., (2020) - Georgian Electricity Balance in the Years of State Independence (1989-2019). Globalization and Business, N-9 2020.

Narmania, D., (2020). Coronomics and Socio-Economic of the Energy Sector.

Natural Resources of Georgia (2015) (2 vol.). Publishing House of the Georgian National Academy of Sciences.

Papava, V., (2020). - Proceedings of the Third National Scientific Conference on the Reflection of the Coronomics in Economic Science and Economic Policy. Tbilisi 2020

Tavadze, G., Kavtaradze, I., Chomakhidze, D., Menabde D., (2006). Energy Regulation: Theory and Practice. Tbilisi. 